\title{
Necesidades formativas del profesorado de Secundaria para la implementación de experiencias gamificadas en STEM
}

\section{Secondary Teachers Training Needs to Implement Gamified Experiences in STEM}

\author{
Mercedes Fuentes Hurtado \\ Universitat Rovira i Virgili, Tarragona, España \\ mariadelasmercedes.fuentes@ estudiants.urv.cat \\ Juan González Martínez \\ Programa Serra Húnter. Universitat de Girona, Girona, España \\ juan.gonzalez@udg.edu
}

\begin{abstract}
Resumen
La formación de ciudadanos competentes tecnológicamente es uno de los retos a los que los docentes de Secundaria se enfrentan hoy en los países desarrollados, donde se espera una creciente demanda de profesionales del ámbito científico-tecnológico (Convert y Gugenheim, 2005), pero se observa desinterés por esta rama del conocimiento (Marbà y Márquez, 2010). De ahí parte la iniciativa STEM (Science, Technology, Engineering \& Maths) (Stohlmann, Moore y Roehrig, 2012) que promueve el estudio integrado de estas materias.

Buscando la excelencia académica por medio de metodologías que motiven al alumnado, la gamificación presenta características suficientes para ser considerada un elemento de mejora del proceso de enseñanza-aprendizaje.

Este artículo presenta, mediante un análisis documental, los tres saberes (contenidos, pedagogía y tecnología), que siguiendo el modelo pedagógico TPACK (Mishra y Koehler, 2006) deben ser desarrollados por los docentes de STEM para implementar experiencias gamificadas.
\end{abstract}

Palabras clave

Formación docente, gamificación, Secundaria, STEM, TPACK.

\footnotetext{
Abstract

Training citizens to develop their technical competence is a great challenge for Secondary Education teachers in developed countries, where demand for scientific and technical professionals is expected to increase (Convert y Gugenheim, 2005), but students show a lack of interest in this field (Marbà y Márquez, 2010). As a consequence of this, STEM emerges (Science, Technology, Engineering \& Maths) (Stohlmann, Moore y Roehrig, 2012) to promotes integrated learning.

Looking for academic excellence through methodologies that motivate students, gamification has characteristics to be considered as an important element in order to improve teaching-learning process.

This article shows, by means of a documentary analysis, the three knowledge areas (contents, pedagogy and technology) that follow TPACK model (Mishra y Koehler, 2006) and should be developed by STEM teachers to implement gamified experiences.

Key words

Gamification, Secondary, STEM, teachers training, TPACK.
}

\section{Introducción.}

La Sociedad del Conocimiento (SC) en la que vivimos se encuentra en constante cambio y esto se refleja también en las instituciones educativas. El nuevo paradigma educativo exige que se transforme la figura tradicional del profesorado como mero transmisor de conocimientos (Von Garnier, 2010) y que, como consecuencia de ello, este asuma cada vez más el rol de guía, acompañante o facilitador del aprendizaje 
(Gisbert, González y Esteve, 2016; Molas y Rosselló, 2010) de un alumnado cuyo interés y cuya curiosidad por el mundo que le rodea deben estimularse, en un planteamiento más acorde con las teorías constructivistas (Piaget, 1967; Vygotski, 1934).

En este escenario de enfoque, la profesionalidad de los docentes siempre está en el punto de mira cuando se habla de calidad educativa. Y, en ese sentido, aun dejando al margen los problemas que se dan en las aulas y que pueden llegar a desmotivar al profesorado, con el consecuente empeoramiento de su labor educativa (Esteve, 2003), son abundantes los informes que se vienen presentando por organismos internacionales (UNESCO, 2008; MECD, 2014) y que muestran la necesidad global de mejorar la formación del profesorado (Campos, 2012; Bozu e Imbernon, 2016). Sin embargo, esta responsabilidad recae en muchas ocasiones en los propios docentes (Olmedilla, 2006; Arza, 2016), quienes no reciben ningún incentivo por mantener sus conocimientos o metodologías didácticas actualizadas con el fin de mejorar el rendimiento académico del alumnado y contribuir a prevenir el fracaso escolar (que en España según los últimos datos del Ministerio de Educación, Cultura y Deporte (2017) alcanza el 20\%), y preparar a los jóvenes para que sean capaces de enfrentarse a los nuevos retos del siglo XXI (Marcelo, 2001; Area, 2010). La resolución de esos retos, en definitiva, será lo que demuestre, en palabras de Esnaola (2009) y Lengeren (2016), que los conocimientos que hayan adquirido son realmente significativos.

Por tanto, en el contexto educativo actual, "el profesorado debe desplegar nuevas habilidades y maneras de plantear las estrategias educativas" (Molas y Roselló, 2010, p. 1) que repercutan positivamente en el proceso de enseñanza aprendizaje. Como consecuencia de ello, González Sanmamed (2009, p. 57) explica que "la enseñanza se ha convertido en una tarea compleja, difícil y problemática" y que la necesidad de formación permanente del profesorado de Secundaria se debe principalmente a la complejidad de las tareas que realiza (Darling-Hammond y Bransford, 2005; Escudero, 2006; Esteve, 2001, 2003; Esteve, Franco y Vera, 1995; Marcelo, 2007; Montero, 2004; Gimeno, 2002). Entre ellas, destaca luchar contra el fracaso o el abandono escolar prematuro (Tarabini, Curran, Montes y Parcerisa, 2015) y, en definitiva, mejorar la calidad de los aprendizajes. Ambos aspectos, por su parte, nos llevan a la necesidad de fomentar la motivación del alumnado, lo que resulta un elemento propiciador del aprendizaje como apunta Lozano (2003, p.45): "cuando un alumno está fuertemente motivado todo su esfuerzo y personalidad se orienta hacia el logro de una determinada meta, empleando para ello todos sus recursos".

Por ello, resulta imprescindible que los profesionales de la educación reciban, a su vez, una formación innovadora y actualizada (Marcelo, 2001; Tribó, 2008; González Sanmamed, 2009; Angelini y García-Carbonell, 2015) que repercuta directamente en la calidad de la enseñanza $\mathrm{y}$, por tanto, en la mejora de los resultados del alumnado (Esteve, 1987; Bona, 2015). Esta necesidad de búsqueda de la excelencia del profesorado también ha saltado a la opinión pública; y así, por ejemplo, Marina (2015) apuesta por el fortalecimiento de la formación docente incluyendo controles periódicos de conocimientos y aptitudes. A su vez, ello coincide con las palabras de Olmedilla (2006, p. 3), quien afirma que "los programas eficaces de formación del personal docente pueden marcar una diferencia importante en los logros de aprendizaje de los estudiantes". Son estudiantes que, por su parte, reconocen sentirse frecuentemente

Necesidades formativas del profesorado de Secundaria para la implementación de experiencias gamificadas en STEM. Mercedes Fuentes Hurtado y Juan González Martínez.

Página 2 de 25 
desmotivados, sobre todo en la etapa de Secundaria, y en especial en las asignaturas del ámbito científico tecnológico (Vázquez y Manassero, 2009; Marbà y Márquez, 2010) alegando razones como la poca o ninguna vinculación entre su realidad y los contenidos que están obligados a estudiar en las diferentes asignaturas.

En otro orden de cosas, la fragmentación de los conocimientos que impone la separación de las materias viene siendo cada vez más cuestionada, como lo muestran las modificaciones introducidas en el sistema educativo español por la Ley 8/2013, de 9 de diciembre, para la mejora de la calidad educativa (LOMCE), que se alinea así con un cambio de paradigma educativo en el que los contenidos se enseñen de manera transversal potenciando la motivación del alumnado por medio de metodologías activas. Esa es una de las claves para, precisamente, conectar los contenidos con el mundo y, por tanto, con la realidad del propio alumnado (Von Garnier, 2010). En el caso concreto del ámbito científico y tecnológico, este cambio de paradigma se materializa en la iniciativa STEM (Duque, Celis y Camaño, 2011), acrónimo de las asignaturas por sus nombres en inglés (Science, Technology, Engineering \& Maths), que nació con el objetivo de favorecer el aprendizaje de las materias del ámbito científico y tecnológico por medio de la integración de los contenidos. Con ello, STEM propone acercar el conocimiento de este ámbito a la realidad del alumnado con un triple objetivo encadenado: (1) vencer la desmotivación creándole interés por el mundo en el que vive, (2) repercutir directamente en su rendimiento académico (Peña y Madrid, 2015) y (3) fomentar las vocaciones científicas (Convert y Gugenheim, 2005; Vázquez y Manassero, 2009; Stohlmann, Moore y Roehrig, 2012). La iniciativa STEM se está desarrollando en distintos países de diversas formas (Prieto y Chrobak, 2012) y en España, aunque el término STEM no aparece explícitamente en la LOMCE, sí se ha incluido una nueva competencia clave, la competencia matemática y competencias básicas en ciencia y tecnología (CMCT), que ha de ser desarrollada por todo el alumnado.

Con todo, este nuevo paradigma educativo descrito por Von Garnier (2010) y concretado para el ámbito STEM requiere de nuevas metodologías didácticas que conecten con el alumnado digital (Gallardo, 2012) que puebla hoy las aulas y con las que se mejoren los resultados académicos incentivando la motivación del alumnado. Una de las muchas metodologías adoptadas por los docentes que tienen como objetivo, como acabamos de decir, la mejora de los resultados académicos buscando la motivación del alumnado, es la gamificación, que ha gozado de abundante predicamento en los últimos años (Kapp, 2012; Sánchez-Aparicio, 2014; Marín, 2015; Rodríguez y Santiago, 2015; González y Fuentes, 2016) y que se apoya en el conocimiento de que los juegos pueden despertar cualquier emoción (Herranz y Colomo-Palacios, 2012; Werbach, 2012). Tal y como se ha hecho con éxito en otros contextos no lúdicos (Cortizo Pérez et al., 2011; Ovallos, Villalobos, De la Hoz, Maldonado, 2016), cabe, por tanto, pensar que introducir elementos del juego en el contexto educativo, es decir, implementar una metodología gamificada (Foncubierta y Rodríguez, 2006; Zichermann y Cunningham, 2011; Kapp, 2012; Díaz y Troyano, 2013) puede contribuir a la mejora de los aprendizajes. Con ello, aprovecharíamos ciertas bondades relacionadas con la estimulación de la curiosidad, el disfrute, la satisfacción y la implicación (Hamari, Koivisto y Sarsa, 2014) al servicio de nuevos y mejores aprendizajes. Por último, esta metodología gamificada de la que hablamos permite ser digitalizada (o tecnologizada) fácilmente gracias a numerosas aplicaciones a disposición del docente (Classcraft, ClassBadges, Class Dojo, Kahoot) para la

Necesidades formativas del profesorado de Secundaria para la implementación de experiencias gamificadas en STEM. Mercedes Fuentes Hurtado y Juan González Martínez.

Página 3 de 25 
consecución de los objetivos didácticos y para la mejora del rendimiento académico por medio de la motivación del alumnado (Murua, 2013; Marín, 2015).

Ahora bien, ¿está el profesorado en disposición de hacer frente a este reto con su perfil competencial actual? ¿Atesora los saberes disciplinares, pedagógicas y tecnológicas necesarias para ello? Sin duda, la revisión de la literatura evidencia a priori que el profesorado se ve en la necesidad de aplicar nuevas metodologías que favorezcan el aprendizaje significativo por medio del uso de las Tecnologías de la Información y la Comunicación (TIC) (Almerich, Suárez-Rodríguez, Belloch y Bo, 2011; Esteve-Mon, Gisbert-Cervera y Lázaro-Cantabrana, 2016). Concretando para el ámbito científicotecnológico y buscando la estimulación de la motivación de los estudiantes para así provocarles interés por el mundo que les rodea y por el conocimiento en general, en este artículo ponemos el foco en la gamificación digitalizada de STEM (González y Fuentes, 2016) y analizamos cuáles son las necesidades formativas del profesorado que desee introducir metodologías gamificadas enriquecidas con TIC en el contexto de la Educación Secundaria Obligatoria (ESO) del sistema educativo español para el ámbito de STEM, buscando siempre la calidad educativa por medio del aprendizaje significativo de los contenidos curriculares y despertando la motivación en el alumnado. Como veremos detenidamente en los apartados siguientes, estas necesidades formativas, que parten del perfil competencial necesario para desarrollar experiencias de aprendizaje gamificadas en el ámbito STEM, se concretan en tres saberes: contenidos, pedagogía y TIC, que se corresponden con los tres ejes fundamentales de conocimientos que propone el modelo pedagógico TPACK para la formación del profesorado descrito por Mishra y Koehler (2006) y que tomaremos como fundamento teórico en esta investigación a la hora de definir la formación docente óptima.

Por tanto, el objetivo de este artículo es, precisamente, justificar a partir de la revisión de la literatura cuáles son los contenidos y las destrezas, dentro de un marco general de competencia digital docente, en que deberíamos formar a los docentes para que diseñen e implementen experiencias de aprendizaje gamificadas para el ámbito STEM.

\section{Método.}

Este artículo forma parte de la primera fase de una investigación más extensa que sigue la metodología de Investigación Basada en Diseño, denominada DBR por sus siglas en inglés (Plomp, 2013; Reeves, Herrington y Oliver, 2005; De Benito y Salinas, 2016), orientada al diseño y a la validación de una estrategia de formación en gamificación para el ámbito STEM para el profesorado en activo de Educación Secundaria. Dentro de la primera fase dedicada al diagnóstico y a la fundamentación, se ha realizado un análisis documental para recopilar, estudiar y analizar a partir de las buenas prácticas recabadas en la literatura cuáles son los contenidos y las destrezas, dentro de un marco general de competencia digital docente, en que deberíamos formar a los docentes para que diseñen e implementen experiencias de aprendizaje gamificadas para el ámbito STEM.

En concreto, el análisis documental que proponemos queda operativizado según las recomendaciones descritas por Bisquerra (2009), que se resumen en los siguientes puntos:

- Rastreo e inventario de los documentos disponibles.

- Clasificación de los documentos identificados.

- Selección de los documentos más pertinentes para la investigación.

- Lectura en profundidad del contenido.

Necesidades formativas del profesorado de Secundaria para la implementación de experiencias gamificadas en STEM. Mercedes Fuentes Hurtado y Juan González Martínez.

Página 4 de 25 
- Lectura cruzada y comparativa de los documentos para construir una síntesis.

\subsection{Rastreo e inventario de los documentos disponibles}

Ese análisis documental parte de una selección de textos de la literatura del ámbito llevada a cabo por medio de búsquedas selectivas cruzadas de documentos a partir de las palabras clave relevantes en los repositorios más accesibles y sensibles a la temática (WoK, iCercador, Google Schoolar, ERIC, Scopus), lo cual nos ha permitido constituir una batería de textos con los que armar la presente reflexión sobre STEM y gamificación para la educación tanto de forma disgregada como documentos que relacionan ambos conceptos. Tras las lecturas iniciales, se procedió a armar un mapa conceptual, a partir del cual se produjo la redacción de esta reflexión, al servicio del objetivo que hemos enunciado anteriormente, lo que viene a suponer una primera revisión, o practical screen (Okoli y Schabram, 2010).

\subsection{Clasificación, selección y síntesis de los documentos consultados}

Los documentos que finalmente forman parte del corpus literario analizado (ver Tabla 1) se han clasificado en tres grupos:

- Documentos específicamente relativos al ámbito STEM y la enseñanza integrada de las disciplinas del ámbito científico y tecnológico.

- Documentos relativos a la gamificación en el ámbito educativo formal en general.

- Documentos relativos a experiencias docentes gamificadas puestas en práctica en diferentes escenarios educativos en Primaria, Secundaria y Universidad para STEM u otras disciplinas, siempre que se hayan considerado extrapolables al ámbito STEM.

Tabla 1.

Número de documentos consultados por temática.

\begin{tabular}{|l|c|}
\hline \multicolumn{1}{|c|}{ Temática } & \multicolumn{1}{|c|}{$\begin{array}{c}\mathbf{N}^{\mathbf{0}} \text { de documentos } \\
\text { consultados }\end{array}$} \\
\hline $\begin{array}{l}\text { Ámbito STEM y enseñanzas } \\
\text { integradas }\end{array}$ & 9 \\
\hline Gamificación en educación & 23 \\
\hline Gamificación en STEM & 24 \\
\hline
\end{tabular}

De entre los tres grupos de documentos clasificados, es el último el que reviste mayor interés para esta investigación, ya que se focaliza en la aplicación de la gamificación en el ámbito STEM y engloba los documentos que se han priorizado en este estudio por estar directamente relacionados con el objetivo que se persigue.

\subsection{Evaluación cualitativa y análisis}

A partir de aquí, se procedió al análisis cualitativo en sí mismo, que considera la evaluación cualitativa y la extracción de los datos relevantes (Okoli y Schabram, 2010). Para facilitarlo se procedió a una primera categorización de la información relevante en

Necesidades formativas del profesorado de Secundaria para la implementación de experiencias gamificadas en STEM. Mercedes Fuentes Hurtado y Juan González Martínez.

Página 5 de 25 
tres ámbitos, tomados del modelo TPACK (Mishra y Koeler, 2006, 2008). En este sentido, si tuviéramos que enumerar las características de un profesional de la enseñanza en la actualidad, diríamos que los docentes deben ser buenos conocedores de la materia que imparten en cuanto a contenidos se refiere, disponer de conocimientos pedagógicos adecuados y tener unas determinadas características personales que le habiliten para la docencia, según Zabalza (2003) y Salinas, De Benito y Lizana (2014). Además, si partimos de las características de la Sociedad del Conocimiento y del tipo de alumnado digital actual (Gallardo, 2012), habría que añadir una cualidad más al profesorado de hoy en día y esta es la necesidad de que los docentes sean competentes digitalmente (Del Moral y Villalustre, 2010; Gisbert, González y Esteve, 2016; Pico, 2013; Tribó, 2008). Por tanto, el docente actual debe disponer de conocimientos en contenidos, pedagogía y TIC que le hagan capaz de enfrentarse a la compleja tarea de facilitar el aprendizaje al alumnado digital. Estos tres saberes son los que se consideran primordiales en el modelo pedagógico de formación docente TPACK introducido por Mishra y Koehler $(2006 ; 2008)$ y que se presentan en la figura 1.

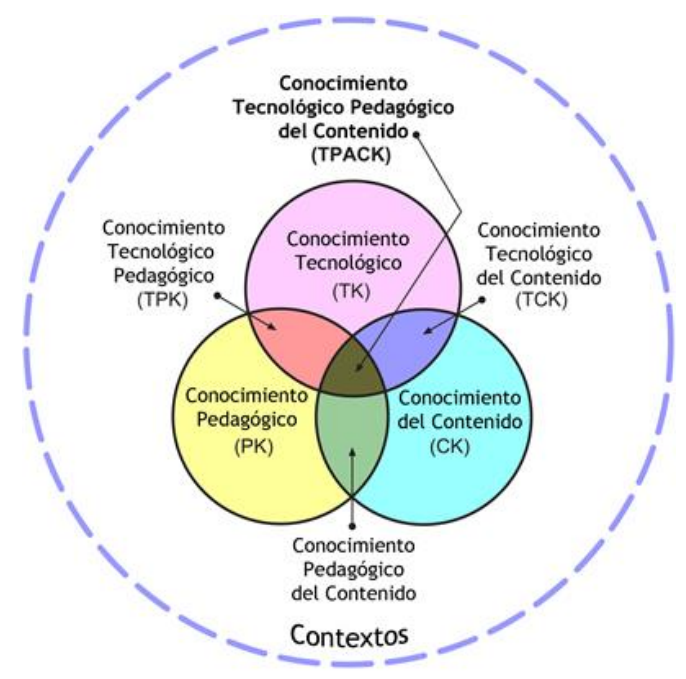

Figura 1. Diagrama del modelo pedagógico TPACK. (Adaptado de Salinas, De Benito y Lizana, 2014)

A partir del esquema general (adaptado de Salinas, De Benito y Lizana, 2014) de la figura 1 y siguiendo, por tanto, el modelo TPACK, los conocimientos fundamentales que debe adquirir un docente del ámbito STEM que pretenda introducir metodologías gamificadas son los siguientes:

- Conocimiento del Contenido (CK): conocer en profundidad los currículos de las materias del ámbito STEM que en el sistema educativo español se corresponden con Biología y Geología, Física, Matemáticas y Tecnología y saber impartir los contenidos comunes de dichas materias de manera integrada.

- Conocimiento Pedagógico (PK): conocer y saber aplicar metodologías gamificadas que faciliten el proceso de enseñanza aprendizaje buscando la motivación del alumnado, su implicación y su interés por el conocimiento.

Necesidades formativas del profesorado de Secundaria para la implementación de experiencias gamificadas en STEM. Mercedes Fuentes Hurtado y Juan González Martínez. 
- Conocimiento Tecnológico (TK): ser competente digitalmente y saber usar las TIC más adecuadas para llevar a cabo la implementación en el aula de metodologías gamificadas.

A continuación, se exponen los datos obtenidos a este respecto partiendo de estas tres categorías de análisis.

\section{Resultados.}

\subsection{Conocimiento del contenido del docente de STEM}

En el sistema educativo español, y teniendo en cuenta las leyes educativas que, aunque cuestionadas, siguen vigentes en la actualidad (en esencia, la Ley 2/2006, de 3 de mayo, de educación (LOE) con las modificaciones introducidas por la Ley 8/2013, de 9 de diciembre, para la mejora de la calidad educativa (LOMCE)), la integración de las materias del ámbito STEM consiste en englobar los contenidos de las asignaturas de Biología y Geología (ByG), Matemáticas (Mat), Tecnología (Tec) y Física y Química (FyQ). Todas ellas son obligatorias en la etapa de la Educación Secundaria Obligatoria, (ESO, de los 12 a los 16 años), a excepción de Tecnología que, aunque se imparte como asignatura obligatoria en comunidades como Andalucía y Madrid, se oferta como optativa en todos los cursos de la ESO y es troncal en el 2. ${ }^{\circ}$ ciclo. Estas materias del ámbito científico tecnológico se distribuyen en bloques de contenidos cuyas similitudes permiten establecer una correspondencia entre los bloques temáticos de las distintas asignaturas tal y como se muestra en la Tabla 2.

Tabla 2.

Elaboración propia. Relación de bloques de contenidos de las asignaturas STEM según la LOMCE en la ESO.

\begin{tabular}{|c|c|c|c|c|c|}
\hline & Física y Química ( $2^{\circ}$ y $3^{\circ}$ ESO) & Biología y Geología & $\left(10,2^{\circ}\right.$ y $3^{\circ}$ ESO & & Matemáticas \\
\hline \multirow{5}{*}{ 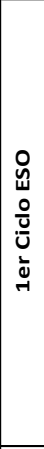 } & Bloque 1. La actividad científica & $\begin{array}{l}\text { Bloque 1. Habilidades, destrezas y estrategias. } \\
\text { Metodología científica. } \\
\text { Bloque 3. La biodiversidad en el planeta Tierra } \\
\text { Bloque 7. Proyecto de investigación }\end{array}$ & $\begin{array}{l}\text { Bloque 1. Proceso de resolución de problemas } \\
\text { tecnológicos } \\
\text { Bloque 2. Expresión y comunicación técnica }\end{array}$ & \multirow{5}{*}{ 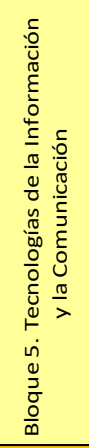 } & \multirow{5}{*}{ 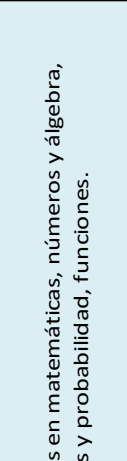 } \\
\hline & Bloque 2. La materia & Bloque 2. La Tierra en el universo & Bloque 3. Materiales de uso técnico & & \\
\hline & Bloque 3. Los cambios & $\begin{array}{l}\text { Bloque 2. La Tierra en el universo } \\
\text { Bloque 4. Las personas y la salud. Promoción de la } \\
\text { salud }\end{array}$ & Bloque 3. Materiales de uso técnico & & \\
\hline & $\begin{array}{l}\text { Bloque 4. El movimiento y las } \\
\text { fuerzas }\end{array}$ & $\begin{array}{l}\text { Bloque 2. La Tierra en el universo } \\
\text { Bloque 5. El relieve terrestre y su evolución }\end{array}$ & $\begin{array}{l}\text { Bloque 4. Estructuras y mecanismos: máquinas } \\
\text { y sistemas }\end{array}$ & & \\
\hline & Bloque 5. Energía & $\begin{array}{l}\text { Bloque 5. El relieve terrestre y su evolución } \\
\text { Bloque 6. Los ecosistemas }\end{array}$ & $\begin{array}{l}\text { Bloque 4. Estructuras y mecanismos: máquinas } \\
\text { y sistemas }\end{array}$ & & \\
\hline \multirow{6}{*}{ 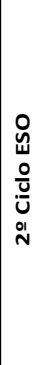 } & Física y Química (4 ESO) & $\begin{array}{|cc|}\text { Biología y Geología } \quad \text { (40 ESO) } \\
\end{array}$ & Tecnología $\quad$ (40ESO) & \multirow{6}{*}{ 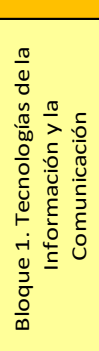 } & $\varrho_{0}^{n} \stackrel{n}{0}$ \\
\hline & Bloque 1. La actividad científica & $\begin{array}{l}\text { Bloque 1. La evolución de la vida } \\
\text { Bloque 4. Proyecto de investigación }\end{array}$ & Bloque 6. Tecnología y sociedad & & 党 \\
\hline & Bloque 2. La materia & Bloque 3. Ecología y medio ambiente & Bloque 3. Electrónica & & \\
\hline & Bloque 3. Los cambios & $\begin{array}{l}\text { Bloque 2. La dinámica de la Tierra } \\
\text { Bloque 3. Ecología y medio ambiente }\end{array}$ & Bloque 2. Instalaciones en viviendas & & 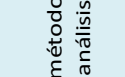 \\
\hline & $\begin{array}{l}\text { Bloque 4. El movimiento y las } \\
\text { fuerzas }\end{array}$ & Bloque 2. La dinámica de la Tierra & $\begin{array}{l}\text { Bloque 4. Control y robótica } \\
\text { Bloque 5. Neumática e hidráulica }\end{array}$ & & ò \\
\hline & Bloque 5. La energía & Bloque 3. Ecología y medio ambiente & $\begin{array}{l}\text { Bloque 3. Electrónica } \\
\text { Bloque 4. Control y robótica }\end{array}$ & & $\overline{0}$ \\
\hline
\end{tabular}

Necesidades formativas del profesorado de Secundaria para la implementación de experiencias gamificadas en STEM. Mercedes Fuentes Hurtado y Juan González Martínez. 
Como decíamos, en esta Tabla 2 se relacionan los bloques de contenidos establecidos por la LOMCE para las cuatro asignaturas del ámbito STEM en los dos ciclos de la etapa de la ESO teniendo en cuenta los contenidos, los criterios de evaluación y los estándares de aprendizaje que propone dicha ley. El primer ciclo de la etapa corresponde a los cursos de $1 .^{\circ}, 2 .^{\circ}$ y $3 .^{\circ}$ y el segundo ciclo corresponde a $4 .^{\circ}$ de la ESO. Las cuatro materias del ámbito aparecen separadas por columnas y por ciclo, así la tabla se divide en dos partes, en la parte superior se encuentra el $1{ }^{\mathrm{er}}$ ciclo de la ESO y la parte inferior el 2..$^{\circ}$ ciclo. Los bloques de contenidos de cada materia que se muestran por columnas se relacionan entre sí horizontalmente por filas. De esta manera y tomando como ejemplo la primera fila del $1{ }^{\mathrm{er}}$ ciclo de la ESO, se establece que el bloque 1 de FyQ está íntimamente relacionado con los bloques 1, 3 y 7 de ByG y los bloques 1 y 2 de Tec, además de con el bloque $5 \mathrm{de}$ Tec, que es transversal al tratarse del conocimiento de Tecnologías de la Información y la Comunicación (TIC) y que debe desarrollarse a lo largo de todo el primer ciclo de la ESO. Esto significa que todos estos bloques de contenidos (bloque 1 de FyQ; 1, 3 y 7 de ByG; 1, 2 y 5 de Tec) serían fácilmente integrables con el apoyo, además, de la asignatura de Matemáticas (Mat) que resulta transversal a las otras tres materias en toda la etapa de la ESO, ya que los fundamentos o procedimientos matemáticos que se requieran dependerán de cada tarea o ejercicio planteado.

Aunque los bloques de contenidos de las diferentes materias no coinciden al cien por cien, sí es posible establecer vínculos entre ellos que permiten combinarlos de manera apropiada para que puedan impartirse conjuntamente. Si nos detenemos en la primera fila de la tabla donde, como decíamos, se establece una relación entre los bloques 1 de FyQ, 1, 3 y 7 de ByG y 1 y 2 de Tec, (además de con el bloque 5 de Tec referente a las TIC y Matemáticas que, como se ha explicado, resultan transversales) observamos que el fundamento de los bloques, según se describe en la LOMCE, es conocer las etapas del método científico (bloque 1 de FyQ, bloques 1 y 7 de ByG) que coinciden con las etapas del proceso de resolución de problemas tecnológicos (bloque $1 \mathrm{de}$ Tec) y que podrían llevarse a la práctica, para fomentar el aprendizaje significativo, mediante la investigación o estudio de la biodiversidad en el planeta Tierra (bloque 3 de ByG) para lo cual será necesario un proceso de investigación usando las TIC (bloque 5 de Tec), comprender y manejar los datos obtenidos (Mat) que finalmente deberían ser presentados junto con las conclusiones y soluciones propuestas debidamente argumentadas (bloque 2 de Tec de expresión y comunicación técnica).

Del mismo modo, si nos fijamos ahora en la cuarta fila, donde se relacionan el bloque 4 de FyQ, los bloques 2 y 5 de ByG, el bloque 4 de Tec y, de nuevo, aparecen de manera transversal el bloque 5 de Tec y las Matemáticas, observamos que los fundamentos físicos que rigen la descripción de los movimientos y las fuerzas, contenidos del bloque 4 de FyQ, son los mismos principios que han de tenerse en cuenta en el diseño y construcción de estructuras y mecanismos (bloque $4 \mathrm{de}$ Tec) ya que las estructuras se ven sometidas a fuerzas y los mecanismos transforman o transmiten el movimiento de unos sistemas a otros en una máquina. Igualmente a como ocurre en las máquinas y las estructuras artificiales que se estudian en Tecnología, la Tierra está formada por estructuras naturales que se ven sometidas a fuerzas que provocan movimientos en las distintas capas de la Tierra originando las modificaciones en el relieve terrestre (bloque 5 de ByG). Además, la Tierra como planeta se ve sometida a fuerzas gravitatorias que permiten su movimiento dentro del universo como parte del Sistema Solar (bloque 2 de

Necesidades formativas del profesorado de Secundaria para la implementación de experiencias gamificadas en STEM. Mercedes Fuentes Hurtado y Juan González Martínez.

Página 8 de 25 
ByG). Fuerzas y movimientos tanto del relieve terrestre como del planeta en el universo que se rigen por los mismos principios físicos las que comentábamos antes. Para el estudio de estos bloques es obviamente necesario hacer uso de fórmulas matemáticas y manejar distintas unidades que permitan realizar los cálculos necesarios para estimar el valor de las fuerzas y de los distintos parámetros que intervienen en la transmisión y transformación de movimientos (distancias, pesos, etc.). Todo ello con el apoyo de las herramientas TIC necesarias tanto para la realización de cálculos como para lograr una mayor comprensión de los conceptos mediante simulaciones o búsqueda de información en la red. Por fundamentarse en los mismos principios, estos bloques de contenidos de FyQ, Tec, ByG y Mat están íntimamente relacionados y podrían impartirse de manera integrada haciendo que el alumnado comprenda mejor el mundo que le rodea y la conexión entre distintos elementos.

Por su formación básica previa, damos por descontado, que los profesores del ámbito de STEM tienen altos conocimientos de los contenidos que son comunes a las cuatro asignaturas del ámbito y que se basan en los mismos principios físicos y matemáticos, aunque su especialidad sea teóricamente solo una de ellas. Sin embargo, sí es necesario ofrecerles a los docentes de este ámbito una formación que incida en identificar los puntos de conexión de los distintos bloques de contenidos de las materias STEM tal y como aparecen sintetizados en la tabla 2 para la etapa de la ESO. Por tanto, el conocimiento del contenido, al que hace referencia el modelo TPACK, y en el que pretendemos formar en un futuro al profesorado de STEM, se concreta en la búsqueda de las similitudes entre los bloques de contenidos de las cuatro materias del ámbito identificando qué contenidos se pueden impartir de manera integrada y mostrando de forma práctica cómo se articularía ese abordaje conjunto de los contenidos multidisciplinares en el tercer nivel de concreción. Todo ello permite ofrecer un nuevo enfoque a los contenidos curriculares que debe impartir el docente de cualquiera de estas cuatro materias.

\subsection{Conocimiento pedagógico del docente de STEM}

Una vez concretado el nuevo enfoque que debería darse a los contenidos curriculares en el ámbito de STEM, el segundo paso consistiría en analizar las necesidades pedagógicas del profesorado que imparte dichos contenidos en la etapa de la ESO para que lo haga de la manera más satisfactoria posible. Como decíamos, eso pasa por mejorar el proceso de enseñanza-aprendizaje de un alumnado que podemos considerar digital (Gallardo, 2012) por la familiaridad que tiene con las TIC y el uso que hace a diario de dispositivos, aplicaciones, recursos y medios digitales (Sánchez-Aparicio, 2014). Ante este tipo de alumnado, generalmente no funcionan los tradicionales enfoques superficiales de aprendizaje y evaluación basados en la memorización por repetición de hechos o ideas apenas interrelacionados (Maquilón y Hernández, 2011; Mirete, Soro y Maquilón, 2015). Este alumnado, recordamos, se siente además frecuentemente desmotivado por las dificultades que presentan las materias del ámbito STEM (Marbà y Márquez, 2010) y eso le lleva a alejarse de esta rama del conocimiento a medida que avanza en sus estudios (Vázquez y Manassero, 2009; Convert y Gugenheim, 2005). O incluso, puede sentir una profunda desconexión y un claro desinterés por lo académico en general, lo cual puede generar situaciones disruptivas que afectan negativamente al proceso de enseñanza-aprendizaje y a la convivencia en los centros educativos (Muñoz, Pérez, y Martín, 2006) o que finalmente provocan el abandono prematuro de los estudios (Mirete, Soro y Maquilón, 2015; Tarabini, Curran, Montes y Parcerisa, 2015).

Necesidades formativas del profesorado de Secundaria para la implementación de experiencias gamificadas en STEM. Mercedes Fuentes Hurtado y Juan González Martínez.

Página 9 de 25 
Ante el fracaso relativo de los enfoques educativos tradicionales en los contextos actuales, resulta imprescindible un cambio pedagógico que, sin duda, implica la incorporación de metodologías docentes innovadoras que mejoren el proceso de enseñanza-aprendizaje y favorezcan el rendimiento escolar por medio de un aprendizaje significativo. En ese sentido, tal y como se indica en la LOMCE, las metodologías docentes han de permitir el desarrollo de la creatividad y la innovación en el alumnado, dos habilidades que se consideran imprescindibles para los ciudadanos del siglo XXI y que recalca Moreno Marchal (2012, p. 2) afirmando que "de la creatividad y de la innovación dependen la iniciativa empresarial, la investigación, el desarrollo económico, el bienestar social, la competitividad de las organizaciones y también, y no menos importante, el desarrollo personal". Además, una metodología óptima debe buscar la motivación del alumnado despertando su curiosidad y estimulando su conocimiento, haciendo que los estudiantes se sientan satisfechos con su propio aprendizaje y se impliquen en las tareas que realizan. En definitiva, debe hacérseles disfrutar del aprendizaje a la vez que se consigue el objetivo primordial, que no es otro que la mejora de los resultados académicos (Peña y Madrid, 2015). Estas características (creatividad, innovación, motivación, curiosidad, satisfacción), por ejemplo, coinciden en su totalidad con las propias de las metodologías gamificadas (Ocaña et al, 2015, Vega y Cañas, 2014; Albir-Mañanes, 2014; Lizbardo, 2015; Revuelta et al, 2015; VegaMoreno et al, 2016) donde se aplican elementos de la mecánica de juegos a ámbitos de no juego como es, en este caso, el educativo. En palabras de Saorin et al (2015, p. 107), hablando sobre gamificación, "esta tendencia educativa trata de potenciar la motivación, la concentración, el esfuerzo y otros valores comunes a todos los juegos para influir y motivar a los alumnos", consiguiendo así que en el proceso de enseñanza-aprendizaje los estudiantes sean los protagonistas teniendo el control sobre la experiencia lúdica (Barragán et al, 2015) y buscando siempre un aprendizaje experiencial (Lengeren, 2016) y significativo (Peña y Madrid, 2015). Las metodologías gamificadas son avaladas también por Olmo (2015), quien citando a Muñoz Calle (2010), recuerda que el aprendizaje del alumnado se ve potenciado con el empleo adecuado de juegos educativos. Es por todo esto por lo que, aun conociendo otras metodologías que persiguen la mejora del rendimiento académico, y siendo conscientes de que como afirman Urh, Vukovic, Jereb y Pintar (2015) citando a Filsecker y Hickey (2014) la investigación en relación a la gamificación se encuentra en sus albores y, por tanto, no existen pruebas contundentes que afirmen que la gamificación es eficaz en todos los casos, apostamos aquí por la inclusión de metodologías gamificadas que hagan uso de las TIC, ya que consideremos esta metodología provechosa para alcanzar el objetivo de la excelencia académica en STEM, el enriquecimiento del proceso de enseñanzaaprendizaje $y$, en definitiva, una mejora significativa de los resultados del alumnado (González y Fuentes, 2016).

Además de los beneficios descritos que se conjugarían para que el docente consiga una mejora del proceso de enseñanza-aprendizaje, la gamificación resulta una metodología flexible que puede plantearse desde distintos enfoques en cada asignatura según las competencias que se desee potenciar en los alumnos (Molas y Roselló, 2010). Sin embargo, esto obviamente implica una gran dedicación por parte del docente, tal y como nos recuerda Barragán et al $(2015$, p. 710$)$ afirmando que "el proceso de gamificar, al igual que el proceso de virtualizar una asignatura, supone un esfuerzo de planificación y seguimiento, diseño y realimentación constantes y adaptados a cada

Necesidades formativas del profesorado de Secundaria para la implementación de experiencias gamificadas en STEM. Mercedes Fuentes Hurtado y Juan González Martínez.

Página 10 de 25 
asignatura, grupo y curso". Es precisamente este gran requerimiento de esfuerzo y tiempo del docente lo que recalca Brigham (2015) como una de las grandes desventajas de introducir la gamificación en el contexto educativo. El mismo autor va más allá afirmando que la gamificación no garantiza que se facilite el aprendizaje del alumnado en todos los casos, sino que se necesita un buen diseño de actividades relacionadas con objetivos de aprendizaje claros para que la gamificación sea exitosa. Si esto se consigue, el esfuerzo del docente finalmente se verá recompensado por la mejora del rendimiento académico del alumnado.

En el proceso de gamificar el aula, no hay que olvidar elementos como la curiosidad, la competición, el compañerismo y el significado que según Barragán et al (2015), citando a Lazzaro (2004), son considerados las cuatro claves de la diversión y, por tanto, deben aparecer en las metodologías gamificadas en el aula. Estos elementos, a su vez, deberán verse traducidos en elementos reconocibles de la mecánica de juegos por medio de la obtención de puntos, niveles, recompensas, rankings, retos o contenidos desbloqueables (García Velategui, 2015; Olmo, 2015). El docente debe tener en cuenta estos elementos a la hora de implantar experiencias gamificadas, como las denomina García Velategui (2015) quien, coincidiendo con Rodríguez y Santiago (2015), considera que la gamificación no deberían consistir solo en gamificar contenidos a través de videojuegos, sino que también apuesta por incorporar conceptos de gamificación en la propia gestión del aula para aprovechar al máximo los beneficios que esta aporta al alumnado. En ese sentido, de nuevo, Barragán et al (2015) enumeran aspectos como el premio al esfuerzo y al trabajo extra, la penalización cuando hay falta de interés, la propuesta de vías para mejorar y la facilidad para realizar un seguimiento del desempeño académico.

En el caso de STEM, son numerosas las propuestas que integran secuencias gamificadas que avalan los beneficios de introducir la mecánica de juegos en la clase con el objetivo de facilitar al alumnado la adquisición de los contenidos del ámbito y que, además, utilizan las TIC para digitalizar los elementos característicos de los juegos aplicados al ámbito educativo de STEM buscando la motivación del alumnado digital (Ocaña, 2012; Vega y Cañas, 2014; Albir-Mañanes, 2014; Marín, 2015; Lizbardo, 2015; Revuelta et al, 2015; Vega-Moreno et al, 2016). Por tanto, resulta conveniente que los docentes reciban formación específica para desarrollar las habilidades necesarias que permitan no solo introducir juegos educativos con los que se promueve el aprendizaje de los contenidos integrados del ámbito STEM, sino además de gamificar la propia gestión del aula sabiendo implantar en el aula las experiencias gamificadas completas de las que hablábamos antes.

\subsection{Conocimiento tecnológico del docente de STEM}

Nos centramos ahora en la parte final del proceso, en la implantación en el aula de una metodología gamificada, lo que conllevará la toma de decisiones sobre cuáles son los recursos más adecuados y, por tanto, cuáles las TIC más pertinentes. Para ello, debemos tener en cuenta las características del alumnado digital (Gallardo, 2012) de la etapa de la ESO: son ciudadanos de la actual SC tecnificada en prácticamente todos sus campos (Marcelo, 2001). En este sentido, es oportuno traer a colación la necesidad de que el docente desarrolle su propia competencia digital (como ciudadano, y también como docente) de manera que pueda seleccionar y usar las TIC más apropiadas que le permitan desarrollar dicha metodología gamificada con el fin de conseguir sus objetivos didácticos. Así, Gisbert, González y Esteve (2016), afirman que el docente de hoy en

Necesidades formativas del profesorado de Secundaria para la implementación de experiencias gamificadas en STEM. Mercedes Fuentes Hurtado y Juan González Martínez.

Página 11 de 25 
día, al igual que sus alumnos y alumnas, forma parte activa de la SC, y como tal debe desarrollar destrezas y conocimientos que le lleven al ejercicio de su profesión con excelencia sabiendo usar las tecnologías de que disponga para introducirlas con eficacia en el ámbito educativo.

La formación que aquí proponemos debe estar enfocada a mostrar diversas opciones tecnológicas (aplicaciones, recursos, herramientas) que permiten la implementación de experiencias gamificadas. Y ello, lógicamente, implica la mínima capacitación técnica para su configuración y la competencia para su adaptación al ámbito de STEM. Ello, en definitiva, contribuirá a incrementar el uso tecnológico por parte de los docentes y favorecerá el desarrollo de la competencia digital del docente. En definitiva, le permitirá alcanzar un nivel superior en las áreas descritas por el Marco Común de Competencia Digital Docente (MCCDD) (INTEF, 2017): información, comunicación y colaboración, creación de contenidos digitales, seguridad y resolución de problemas. Con esto se busca que el docente, en el ejercicio de su profesión, sea capaz de investigar, seleccionar, configurar y aprender a usar las TIC más apropiadas para crear experiencias gamificadas completas (Garcia Velategui, 2015) en STEM para un determinado grupoclase, donde se gamifiquen tanto los contenidos integrados del ámbito como la gestión del aula por medio de la digitalización de los elementos propios de la mecánica de juegos que, como citábamos antes, son puntos, niveles, retos, contenidos desbloqueables, etc. (García Velategui, 2015; Olmo, 2015).

Con la selección y empleo de las TIC para gamificar los contenidos, se busca facilitar la adquisición de los contenidos integrados de STEM, pues ello favorece que los estudiantes experimenten un aprendizaje vivencial que los haga sentirse más conectados con el conocimiento y entiendan así que tiene relación con sus vidas. En ese sentido, Molas y Roselló (2010), referenciando a Prenski (2005), recuerdan que el uso de las TIC por parte de los estudiantes facilita el proceso de aprendizaje, ya que los convierte en protagonistas de su propio aprendizaje y les permite desarrollar su autonomía resolviendo problemas por ellos mismos en experiencias que les llevan a un aprendizaje vivencial (Levis, 2003). Para la implementación de secuencias gamificadas, el docente dispone de aplicaciones que le permiten gamificar el aula, al mismo tiempo que promueven en el alumnado el desarrollo del pensamiento computacional (Tobón y Cano, 2015; Vega-Moreno et al, 2016) y otras importantes competencias social, lingüística y emprendedora (Moreno et al, 2014). Por ejemplo, aplicaciones como Kahoot (Foncubierta y Rodríguez, 2006) o Zondle (Olmo, 2015), configurables para STEM, permiten establecer rankings basados en preguntas, con los que el profesor puede comprobar si los estudiantes han asimilado los contenidos mediante el juego. Por otro lado, la resolución de problemas en los que se apliquen los contenidos adquiridos puede llevarse a cabo mediante el uso de videojuegos o por medio de la robótica, lo que además permite el desarrollo del pensamiento computacional en los estudiantes por medio del uso de lenguajes de programación lúdicos destinados a tal fin, como Scratch o Lego Mindstorm. En estas experiencias el propio alumnado crea sus videojuegos, donde aplica los conocimientos teóricos adquiridos a la vez que desarrolla capacidades o habilidades de programación. Según Vázquez-Cano y Ferrer (2015), esto les ayudará a tener éxito en cualquier carrera del siglo XXI, ya que el pensamiento computacional promueve el desarrollo de la creatividad, la autonomía para la búsqueda de soluciones, el aprendizaje autónomo, la resolución de problemas y fomenta el trabajo colaborativo,

Necesidades formativas del profesorado de Secundaria para la implementación de experiencias gamificadas en STEM. Mercedes Fuentes Hurtado y Juan González Martínez.

Página 12 de 25 
que puede verse favorecido mediante la compartición de ideas en las redes sociales (Morales Socorro, 2011).

Por otro lado, la gamificación del control del aula se centra principalmente en gamificar las normas de comportamiento del alumnado para favorecer el clima en la clase. En palabras de Rodríguez y Santiago (2015), es el factor que más contribuye a la formación y al rendimiento del alumnado. Aquí no solo entra en consideración si un estudiante es disruptivo o no, sino también lo implicado que esté a la hora de trabajar en grupo, si ofrece ayuda a sus compañeros, si colabora activamente en la realización de las tareas o incluso el mantenimiento de un volumen de voz apropiado durante el trabajo en clase. Comenzando por pequeños gestos como llamar a los alumnos "jugadores" o a los deberes "menús" (Rodríguez y Santiago, 2015) e incorporando las aplicaciones tecnológicas adecuadas, el ambiente de trabajo en el aula mejora. Así, por ejemplo, lo demuestran experiencias docentes gamificadas como la descrita por Tauler (2016) para alumnos de 2. ${ }^{\circ}$ de la ESO en Barcelona por medio de Classcraft. Esta investigación muestra la mejora en el comportamiento del alumnado liberando el aula de momentos disruptivos y fomentando la cooperación entre los estudiantes. En la misma línea, Torres (2016) afirma que esta misma aplicación produce una atractiva atmósfera en la clase que incita a la motivación, y Gómez Triana et al (2015) inciden en su influencia en la mejora del trabajo colaborativo entre los estudiantes. Por su parte, Valle (2016) expone un experimento para la mejora de la conducta de alumnos de Primaria, que podría extrapolarse a Secundaria, por medio de la economía de fichas, mediante la aplicación E_TOKEN. Gracias a ella, se implican todos los agentes relacionados con el aprendizaje del alumnado (tutores, padres, otros alumnos, etc.) y se les da la posibilidad de acceder a ella con diferentes roles. Similar a esta y también para gestionar las insignias del grupo de clase, existen ClassBadges (Manzanares Guillén, 2014) u otras aplicaciones como ClassDojo (García Velategui, 2015; Murua-Cuesta, 2013), que permiten además al docente enviar informes de comportamiento a alumnos y padres. Todo ello, en definitiva, es una pequeña muestra de las muchas posibilidades tecnológicas existentes y sobre las que habrá que formar a los docentes.

Y, por otro lado, para la implantación de experiencias gamificadas en el aula, es importante señalar que, además de los dispositivos tecnológicos de los que disponen generalmente los centros (ordenadores, portátiles, PDI), se pueden emplear también, los dispositivos con los que cuentan los propios estudiantes (smartphones, tablets, etc.), quienes se sumarían de esta manera a la tendencia "BYOD" (Bring Your Own Device) descrita por Saorin et al. (2015).

Resulta obvio, como se ha visto, que para que el docente sea capaz de crear experiencias gamificadas valiéndose de aplicaciones que necesitan ser configuradas y adaptadas al propio ámbito de STEM, es necesario que dichos docentes continúen desarrollando su competencia digital en las áreas descritas por el Marco de Competencia Digital Docente, y esto es lo que se pretende conseguir con esta tercera pata de la formación que aquí se plantea para conseguir la excelencia profesional del docente en el ámbito educativo de STEM.

\section{Discusión y conclusiones}

Los nuevos retos a los que se enfrenta el sistema educativo (y, por ende, el profesorado que tiene la labor de formar a estudiantes que serán los protagonistas del desarrollo de

Necesidades formativas del profesorado de Secundaria para la implementación de experiencias gamificadas en STEM. Mercedes Fuentes Hurtado y Juan González Martínez.

Página 13 de 25 
la sociedad del futuro) obligan a que los profesores continúen formándose constantemente (Campos, 2012; Bozu e Imbernon, 2016) para seguir desarrollando competencias imprescindibles en la Sociedad del Conocimiento (Gisbert, González y Esteve, 2016). No pocos informes oficiales inciden en esta necesidad de formación del profesorado (UNESCO, 2008; MECD, 2014) y es en lo que nos basamos en esta investigación, con especial interés en un ámbito concreto, el STEM (Duque, Celis y Camaño, 2011). Por su relevancia en la actualidad (Convert y Gugenheim, 2005; Prieto y Chrobak, 2012) y por presentar gran dificultad en general para el alumnado de Secundaria (Marbà y Márquez, 2010), debe ser promocionado y enseñado desde un nuevo enfoque educativo que permita integrar los contenidos de las materias del ámbito y conectarlos con la realidad del alumnado (Von Garnier, 2010) favoreciendo el aprendizaje significativo y el desarrollo de habilidades que le permitan enfrentar los retos del siglo XXI (Area, 2010).

En este contexto, y sabiendo que en el nuevo paradigma educativo que se nos plantea son necesarias nuevas metodologías que conecten con el alumnado digital de hoy en día (Gallardo, 2012), consideramos que la gamificación educativa cuenta con características suficientes (fomento de la motivación, implicación y satisfacción por el aprendizaje) (Saorin, 2015) para ser un elemento por incluir en las metodologías docentes cuando se busca mejorar el proceso de enseñanza-aprendizaje. Es indudable que la investigación en gamificación está en sus inicios (Urh, Vukovic, Jereb y Pintar, 2015); y, además, existen voces que recuerdan la dificultad que supone implementar metodologías gamificadas (Barragán, 2015) que tienen el riesgo de fracasar si no están bien planificadas o ligadas a objetivos didácticos concretos, y que, además, gamificar requiere un gran consumo de tiempo por parte de los docentes ya que ha de hacerse para un grupo de clase específico (Brigham, 2015). Sin embargo, sus beneficios quedan demostrados en experiencias concretas que se han llevado a cabo y que exponen como resultado que una buena implementación de la gamificación repercute positivamente en el proceso de enseñanza-aprendizaje (Moreno et al, 2014; Torres, 2016; Tauler; 2016). Por la dificultad que entraña introducir la gamificación en el ámbito educativo y el riesgo que supone una mala implementación de las tareas o actividades a gamificar, consideramos que los docentes deben ser formados adecuadamente para desarrollar con éxito experiencias completas (García Velategui, 2015) para el ámbito STEM que, por medio de las TIC, permitan gamificar tanto los contenidos integrados de las materias científico-tecnológicas como la gestión del aula, con el objetivo único de la mejora de la calidad de los aprendizajes. Aislar esos tópicos según el modelo TPACK (Mishra y Koehler, 2006; 2008) es aquello que proponemos como guía para llevar a cabo una futura propuesta de formación docente que incida en los tres ejes que deben desarrollar los docentes STEM que quieran implementar experiencias gamificadas digitalizadas para grupos de Secundaria.

En este sentido, la búsqueda de la mejora de la calidad educativa nos lleva a plantearnos nuevas formas de enseñar (Bona, 2015) en las que el docente debe abandonar el tradicional rol transmisor para convertirse en un guía o facilitador del aprendizaje (Gisbert, González y Esteve, 2016; Molas y Rosselló, 2010). Es necesario, por tanto, proponer nuevos modelos pedagógicos (con sus correspondientes estrategias didácticas) que hagan recuperar al alumnado el interés por el conocimiento y, por tanto, mejoren el proceso de enseñanza-aprendizaje, sobre todo en el ámbito de la ciencia y la tecnología. En ese sentido, lo consideramos fundamental para enfrentar los nuevos retos que se

Necesidades formativas del profesorado de Secundaria para la implementación de experiencias gamificadas en STEM. Mercedes Fuentes Hurtado y Juan González Martínez.

Página 14 de 25 
plantearán en la sociedad del siglo XXI (Marcelo, 2001; Area, 2010) y que los alumnos catalogan de complicado, difícil o desconectado de la realidad (Vázquez y Manassero, 2009; Peña y Madrid, 2015), lo que les lleva en muchas ocasiones a no decantarse por esta rama del conocimiento a medida que avanzan en sus estudios y que genera, a su vez, una crisis de las vocaciones científicas (Convert y Gugenheim, 2005).

El profesorado, como parte fundamental del proceso de enseñanza-aprendizaje, debe hacer frente al reto que supone enseñar en el siglo XXI y garantizar la excelencia académica (González Sanmamed, 2009; Marina, 2015). Para ello, ha de desarrollar competencias disciplinares, pedagógicas y tecnológicas que les permitan implementar en las aulas nuevas metodologías que mejoren el proceso de enseñanza-aprendizaje, sobre todo, en ámbitos que generalmente presentan dificultades para el alumnado como es el científico y tecnológico.

Por otro lado, para fomentar el rendimiento académico y la motivación del alumnado en el ámbito científico y tecnológico, surgió la iniciativa STEM (Stohlmann, Moore y Roehrig, 2012), que promueve el aprendizaje significativo por medio de la integración de los contenidos de las materias que se engloban en dicho ámbito. En el sistema educativo español, en la etapa de la ESO, estas materias se corresponden con Matemáticas, Biología y Geología, Física y Química y Tecnología, cuyos bloques de contenidos, descritos en la ley vigente de educación (LOE modificada por LOMCE), se relacionan tal y como que se muestra en la tabla 2. Estos contenidos, por tanto, han de enseñarse de manera integrada y para ello los docentes requieren de formación específica que les muestre los puntos de conexión entre las distintas materias del ámbito y cómo se articularía esa integración en la práctica.

Además del nuevo enfoque que ha de darse a los contenidos para enseñarlos de manera integrada en el ámbito STEM, el profesor en su labor docente debe adaptarse a los nuevos tiempos y emplear nuevos enfoques pedagógicos alejados de la memorización (Maquilón y Hernández, 2011; Mirete, Soro y Maquilón, 2015) y más orientados a fomentar un aprendizaje significativo que facilite la adquisición de contenidos, favorezca la motivación, la curiosidad, la implicación del estudiante y evite el fracaso escolar o el abandono prematuro de los estudios (Mirete, Soro y Maquilón, 2015; Tarabini, Curran, Montes y Parcerisa, 2015). Estos nuevos enfoques pedagógicos han de ir acompañados por metodologías innovadoras que ayuden a alcanzar esos objetivos a la vez que desarrollan la creatividad del alumnado y le haga disfrutar del aprendizaje. Por ejemplo, la introducción de metodologías gamificadas demuestra la consecución de estos objetivos (Stohlmann, Moore y Roehrig, 2012; Ocaña, 2012; Vega y Cañas, 2014; Albir-Mañanes, 2014; Lizbardo, 2015; Revuelta et al, 2015; Vega-Moreno et al, 2016), pues mejora el proceso de enseñanza-aprendizaje mientras se propicia un ambiente lúdico en el aula en el que el estudiante es protagonista (Barragán et al, 2015). Además de la gamificación de los contenidos, resulta interesante formar al docente para que pueda crear experiencias gamificadas completas (García Velategui, 2015) donde también se gamifique la gestión del aula; todo ello, además, mejorará la convivencia en el aula.

Tanto para la gamificación de los contenidos como de la gestión del aula es necesario digitalizar los elementos característicos de la mecánica de juegos (puntos, rankings, retos, contenidos desbloqueables, etc.) (García Velategui, 2015; Olmo, 2015) por medio de las aplicaciones tecnológicas disponibles. Por tanto, y en última instancia, resulta

Necesidades formativas del profesorado de Secundaria para la implementación de experiencias gamificadas en STEM. Mercedes Fuentes Hurtado y Juan González Martínez.

Página 15 de 25 
imprescindible formar al docente para que desarrolle su competencia digital docente por medio del uso, la configuración y la adaptación de las TIC más adecuadas para que pueda ser capaz de crear las experiencias gamificadas de las que antes hablábamos. A modo de ejemplo, en epígrafes anteriores se han ido enumerado diversas aplicaciones que pueden ser empleadas para la gamificación de contenidos de STEM y la gestión del aula susceptibles de ser utilizadas en un programa de formación docente que se desarrollará y planificará como resultado final de la investigación que con este artículo se inicia y fundamenta.

De esta manera, formando a los docentes de STEM en los tres saberes del modelo TPACK de Mishra y Koehler (2006) conseguiríamos fomentar la excelencia académica de estos desarrollando las competencias disciplinares, pedagógicas y tecnológicas necesarias para hacer frente al reto de implementar experiencias gamificadas en el aula del siglo XXI. Con la integración de los contenidos STEM para impartirlos según un nuevo enfoque mediante la incorporación de experiencias gamificadas enriquecidas con TIC en el aula se podría alcanzar el objetivo último de recuperar la motivación y el interés del alumnado para mejorar el proceso de enseñanza-aprendizaje y, a la vez, fomentar las vocaciones científicas.

Presentación del artículo: 10 de junio de 2017 Fecha de aprobación: 25 de junio Fecha de publicación: 30 de junio

Fuentes Hurtado, M., y González Martínez, J. (2017). Necesidades formativas del profesorado de Secundaria para la implementación de experiencias gamificadas en STEM. RED, Revista de Educación a Distancia, 54. Consultado el (dd/mm/aaaa) en http://www.um.es/ead/red/54

\section{Financiación}

Esta investigación no ha recibido ninguna subvención específica de los organismos de financiación en los sectores públicos, comerciales o sin fines de lucro.

\section{Referencias bibliográficas}

Albir-Mañanes, M. (2014) La robótica aplicada a la asignatura de química de $1^{o}$ de bachillerato (Trabajo fin de máster). UNIR, La Rioja. Recuperado de http://reunir.unir.net/handle/123456789/2716

Almerich, G., Suárez-Rodríguez, J. M., Belloch, C. y Bo, R. M. (2011). Las necesidades formativas del profesorado en TIC: Perfiles formativos elementos de complejidad. RELIEVE. Revista Electrónica de Investigación Y Evaluación Educativa, 17(2), $1-28$.

Angelini, M. L. y García-Carbonell, A. (2015). Percepciones sobre la Integración de Modelos Pedagógicos en la Formación del Profesorado : La Simulación y Juego y el Flipped Classroom. EKS, 16(2), 16-30. Recuperado de 
https://gredos.usal.es/jspui/bitstream/10366/127197/3/AngeliniGarc\%C3\%ADa_Percepciones.pdf

Area, M. (2010). Multialfabetización y bibliotecas en la escuela del siglo XXI. Boletín de La Asociación Andaluza de Bibliotecarios, 64, 5-17. http://doi.org/0213-6333

Arza, J. (2016). Los retos del sistema educativo. Escritura Pública, 98, 70-73. Recuperado de https://dialnet.unirioja.es/servlet/articulo?codigo=5400911

Barragán et al. (2015). Una propuesta para la motivación del alumnado de ingeniería mediante técnicas de gamificación. En Actas de las XXXVI Jornadas de Automática, 2-4 de septiembre de 2015. (pp. 2-4). Bilbao.

Bisquerra, R. (2009). Metodología de la investigación educativa. Madrid: Muralla.

Bona, C. (2015). La nueva educación. Barcelona: Plaza\&Janés.

Bozu, Z. e Imbernon, F. (2016). La profesión docente en momentos de cambios. ¿Qué nos dicen los estudios internacionales. Profesorado. Revista de Currículum Y Formación Del Profesorado, 20 (3), 467-492. Recuperado de https://recyt.fecyt.es/index.php/profesorado/article/view/54607/33236

Brigham, T. J. (2015). An Introduction to Gamification: Adding Game Elements for Engagement. Medical Reference Services Quarterly, 34(4), 471-480. http://doi.org/10.1080/02763869.2015.1082385

Campos, J. A. (2012) (Coord.). La formación del profesorado novel en la Universidad de Barcelona. Barcelona: ICE-Octaedro.

Convert, B. y Gugenheim, F. (2005). Scientific Vocations in Crisis in France: Explanatory Social Developments and Mechanism. European Journal Vocational Training, 35, 12-20.

Cortizo Pérez, J. C., Carrero García, F., Monsalve Piqueras, B., Velasco Collado, A., Díaz del Dedo, L. I., y Pérez Martín, J. (2011). Gamificación y Docencia: Lo que la Universidad tiene que aprender de los Videojuegos. En VIII Jornadas Internacionales de Innovación Universitaria, (pp. 1-8). Recuperado de http://abacus.universidadeuropea.es/bitstream/handle/11268/1750/46_Gamificacio n.pdf?sequence $=2$

Darling-Hammond, L. y Bransford, J. (Eds.) (2005). Preparing Teachers for a Changing World: What Teacher Should Learn and Be Able to Do. New Jersey: Jossey Bass.

De Benito, B. y Salinas, J. M. (2016). La Investigación Basada en Diseño en Tecnología Educativa. Revista Interuniversitaria de Investigación En Tecnología Educativa, O(0), 44-59. http://doi.org/10.6018/riite2016/260631

Necesidades formativas del profesorado de Secundaria para la implementación de experiencias gamificadas en STEM. Mercedes Fuentes Hurtado y Juan González Martínez. 
Del Moral, M. E., y Villalustre, L. (2010). Formación del profesor 2.0: desarrollo de competencias tecnológicas para la escuela 2.0. Magister: Revista Miscelánea de Investigación, 23, 59-70.

Díaz, J., y Troyano, Y. (2013). El potencial de la gamificación en el ámbito educativo. En III Jornadas de innovación docente de la Facultad de Ciencias de la Educación. "La innovación educativa: Respuesta en tiempos de incertidumbre". Universidad de Sevilla. Recuperado de https://fcce.us.es/sites/default/files/docencia/EL\%20POTENCIAL\%20DE\%20LA \%20GAMIFICACI\%C3\%93N\%20APLICADO\%20AL\%20\%C3\%81MBITO\%2 0EDUCATIVO_0.pdf

Duque, M., Celis, J., y Camacho Díaz, A. (2011). Cómo Lograr Alta Calidad En La Educación De Los Ingenieros: Una Visión Sistémica. Revista Educación En Ingeniería, 6(12), 48-60. Recuperado de http://www.educacioneningenieria.org/index.php/edi/article/view/122

Escudero. J. M. (2006). La formación del profesorado y la garantía del derecho a una buena educación para todos. En J. M. Escudero y A. Luis (Eds). La formación del profesorado y la mejora de la educación (pp. 21-54). Barcelona: Octaedro.

Esnaola, G. A. (2009). Videojuetos "teaching tech": pedagogos de la convergenica global. La docilización del pensamiento a través del macrodiscurso cultural y la convergencia tecnológica. Revista Electrónica Teoría de La Educación, 10(1), $112-133$.

Esteve, J.M. (1987). El malestar docente. Barcelona: Laia.

Esteve, J.M. (2001). El profesorado de secundaria: hacia un nuevo perfil profesional para enfrentar los problemas de la educación contemporánea. Revista de la Facultad de Ciencias de la Educación, 3, 15-42

Esteve, J.M. (2003). La tercera revolución educativa. La educación en la sociedad del conocimiento. Barcelona: Paidós Ibérica.

Esteve, J. M., Franco, S. y Vera, J. (1995). Los profesores ante el cambio social. Barcelona: Arthropos.

Esteve-Mon, F. M., Gisbert-Cervera, M., y Lázaro-Cantabrana, J. L. (2016). La competencia digital de los futuros docentes: ¿cómo se ven los actuales estudiantes de educación? Perspectiva Educacional, 55(2), 38-54. http://doi.org/10.4151/07189729-Vol.55-Iss.2-Art.412

Filsecker, M. y Hickey D. T. (2014). A multilevel analysis of the effects of external rewards on elementary students' motivation, engagement and learning in an educational game. Computers \& Education, 75, 136-148.

Necesidades formativas del profesorado de Secundaria para la implementación de experiencias gamificadas en STEM. Mercedes Fuentes Hurtado y Juan González Martínez. 
Foncubierta, J., y Rodríguez, C. (2006). Didáctica de la gamificación en la clase de español. Edinumen, 1-8. Recuperado de http://www.edinumen.es/pdp14/Didactica_Gamificacion_ELE.pdf

Gallardo, E. (2012). Hablemos de estudiantes digitales y no de nativos digitales. UT. Revista de Ciències de l'Educació, 7-21.

García Velategui, A. (2015). Gestión de aula y gamificación. Utilización de elementos del juego para mejorar el clima en el aula (Trabajo fin de grado). Universidad de Cantabria. Recuperado de https://repositorio.unican.es/xmlui/bitstream/handle/10902/7595/Garc\%C3\%ADa VelateguiAlejandro.pdf?sequence $=1$

Gimeno, J. (2002). El futuro de la educación desde su controvertido presente. Revista de Educación, $\mathrm{n}^{\circ}$ extraordinario, 271-292.

Gisbert, M., González, J., y Esteve, F. M. (2016). Competencia digital y competencia digital docente: una panorámica sobre el estado de la cuestión. Revista Interuniversitaria de Investigación En Tecnología Educativa, (Junio), 74-83. http://doi.org/10.6018/RIITE2016/257631

Gómez Triana A. P.et al. (2015). Fortalecimiento del trabajo colaborativo de los estudiantes del curso undécimo A, en el área de contabilidad-informática del colegio colombo japonés de Bogotá mediante la plataforma de ludificación Classcraft (Trabajo de grado). Fundación Universitaria Los Libertadores. Bogotá. Recuperado de http://repository.libertadores.edu.co/handle/11371/256

González, J., y Fuentes, M. (2016). Fundamentos para el uso de estrategias de gamificación como elemento clave para favorecer el aprendizaje en el ámbito de STEM. En R. Roig-Vila (Ed.), EDUcación y TECnología. Propuestas desde la investigación y la innovación educativa (pp. 314-315). Barcelona: Octaedro.

González Sanmamed, M. (2009). Una nueva oportunidad para la formación inicial del profesorado de Educación Secundaria. Revista de Educación, 350, 57-78.

Hamari, J., Koivisto, J., y Sarsa, H. (2014). Does gamification work? - A literature review of empirical studies on gamification. En Proceedings of the Annual Hawaii International Conference on System Sciences, (HICSS), (pp. 3025-3034). Hawaii, http://doi.org/10.1109/HICSS.2014.377

Herranz, E., y Colomo-Palacios, R. (2012). La Gamificación como agente de cambio en la Ingeniería del Software. Revista de Procesos Y Métricas, 9(2), 30-56.

INTEF (2017) Marco común de competencia digital docente. Recuperado de http://educalab.es/documents/10180/12809/Marco+competencia+digital+docente+ 2017/afb07987-1ad6-4b2d-bdc8-58e9faeeccea

Kapp, K. (2012). The Gamification of Learning and Instruction: Game-Based Methods and Strategies for Training and Education. San Francisco: John Wiley \& Sons.

Necesidades formativas del profesorado de Secundaria para la implementación de experiencias gamificadas en STEM. Mercedes Fuentes Hurtado y Juan González Martínez.

Página 19 de 25 
Lazzaro, N. (2004). Why We Play Games: Four Keys to More Emotion Without Story. Recuperado de http://www.xeodesign.com/xeodesign_whyweplaygames.pdf.

Lengeren, B. (2015). AL-KIMIA : aprender química a través de la gamificación de su historia. Recuperado de https://www.researchgate.net/publication/280920958

Levis, D. (2003). Videojuegos en red: Espacios simbólicos de juego y encuentro, 1-14. Recuperado de http://diegolevis.com.ar/secciones/Articulos/videojuegos_Uned2003_VF.pdf

Ley Orgánica de Educación (LOE) (Ley Orgánica 2/2006, 3 de mayo). Boletín Oficial del Estado, nº 106, 2006, 4 mayo.

Ley Orgánica para la Mejora de la Calidad Educativa (LOMCE) (Ley Orgánica 8/2013, 9 de diciembre). Boletín Oficial del Estado, n 295, 2013, 10 diciembre.

Lizbardo, B. (2015). Metodología de aprendizaje INGLOVE. En XV Encuentro Virtual Educa (Perú 2014). Recuperado de http://reposital.cuaed.unam.mx:8080/jspui/handle/123456789/4074

Lozano, A. (2003). Factores personales, familiares y académicos que afectan al fracaso escolar en la Educación Secundaria. Revista Electrónica de Investigación Psicoeducativa Y Psicopedagógica, 1, 43-66. http://dx.doi.org/10.14204/ejrep

Manzanares Guillén, S. (2014). Sistema de acreditación de aprendizajes basado en insignias (badges) en la materia de matemáticas. Claves Para Una Educación Diversa. http://diversidad.murciaeduca.es/publicaciones/claves/doc/smanzanares.pdf

Maquilón, J. J., y Hernández, F. (2011). Influencia de la motivación en el rendimiento académico de los estudiantes de formación profesional. REIFOP, 14(1), 81-100.

Marbà, A. y Màrquez, C. (2010). ¿Qué Opinan Los Estudiantes De Las Clases De Ciencias? Un Estudio Transversal De Sexto De Primaria a Cuarto De ESO. Enseñanza de Las Ciencias, 8, 19-30.

Marcelo, C. (2001). Aprender a enseñar para la Sociedad del Conocimiento. Revista Complutense de Educación, 12(2), 531-593. Recuperado de http://revistas.ucm.es/index.php/RCED/article/viewFile/RCED0101220531A/167 49Re

Marcelo, C. (2007). La formación docente en la sociedad del conocimiento y la información: avances y temas pendientes. Comunicación y Pedagogía: nuevas tecnologías y recursos didácticos, 218, 52-62.

Marín, V. (2015). La Gamificación educativa. Una alternativa para la enseñanza creativa En Digital Education Review, 27. Recuperado de http://revistes.ub.edu/index.php/der/article/view/13433/pdf 
Marina, J.A. (2015). Despertad al diplodocus. Barcelona: Ariel.

MECD. (2014). TALIS 2013. Estudio internacional de la enseñanza y el aprendizaje. Informe español. Madrid.

Ministerio de Educación Cultura y Deporte. (2017). Datos y Cifras. Curso escolar 2016-2017, 37. Recuperado de https://www.mecd.gob.es/servicios-al-ciudadano$\mathrm{mecd} / \mathrm{dms} / \mathrm{mecd} / \mathrm{servicios}-$ al-ciudadano-mecd/estadisticas/educacion/indicadorespublicaciones-sintesis/datos-cifras/Datosycifras1617esp.pdf

Mirete, A.B., Soro, M. y Maquilón, J. J. (2015). Aprendizaje: El fracaso escolar y los enfoques de Del, medidas para la inclusión educativa. REIFOP, 18(3), 183-196. http://dx.doi.org/10.6018/reifop.18.3.239021

Mishra, P. y Koehler M. J. (2006). Technological pedagogical content knowledge: A framework for teacher knowledge. En Teachers College Record, 108 (6), 10171054.

Mishra, P. y Koehler M. J. (2008). Introducing TPCK. En Handbook of Technological Pedagogical Content Knowledge (TPCK) for Educators. Recuperado de http://www.mendeley.com/research/ what-is-technological-pedagogical-contentknowledge-tpack/

Molas, N., y Rosselló, M. (2010). Revolución en las aulas: llegan los profesores del siglo XXI. La introducción de las TIC en las aulas y el nuevo rol docente. DIM: Didáctica, Innovación Y Multimedia, 19, 1-9. Recuperado de http://raco.cat/index.php/DIM/article/view/214711

Montero, L. (2004). La formación del profesorado: de hoy y para mañana. En J. López, M. Sánchez y P. Murillo (Eds.), Cambiar con la sociedad, cambiar la sociedad (pp. 158-169). Sevilla, Secretariado de Publicaciones de la Universidad de Sevilla.

Morales Socorro, C. (2011). El Aprendizaje basado en Proyectos en la Educación Matemática del siglo XXI Cuaderno de bitácora. En 15 Jornadas para el Aprendizaje y la Enseñanza de las Matemáticas del 2011 (15 JAEM) (pp. 1-23). Gijón. Recuperado de http://www.oei.es/historico/noticias/spip.php?article9088

Moreno, P. J., Puentes, C., Ferrándiz, E., Flores, E., y Acosta, M. (2014). Trivianometrics, una competición académica por equipos en el aula de Econometría Financiera. Proyectos de Innovación y Mejora Docente, 1-4. Recuperado de http://rodin.uca.es/xmlui/handle/10498/17406

Moreno Marchal, J. (2012). Metodologías para la innovación. Una experiencia en formación del profesorado universitario. En Congrés Internacional de Docéncia Universitaria i Innovació. Barcelona.

Necesidades formativas del profesorado de Secundaria para la implementación de experiencias gamificadas en STEM. Mercedes Fuentes Hurtado y Juan González Martínez. 
Muñoz, C., Pérez, D. y Martín, E. (2006). ¿Qué penalizan los docentes?: análisis de la disciplina a través de los partes de incidencia. Infancia y Aprendizaje, 29(4), 423435. Recuperado de http://dx.doi.org/10.1174/021037006778849477

Muñoz Calle, J.M. (2010). Juegos educativos. FyQ formulación. Eureka Enseñ. Divul. Cien., 7(2), 559-565.

Murua-Cuesta, E. (2013). Análisis de la Gamificación como concepto aplicable en el proceso de enseñanza-aprendizaje de las matemáticas en $4^{\circ}$ de ESO. (Trabajo fin de máster) En Re-UNIR Repositorio Digital. Recuperado de http://reunir.unir.net/handle/123456789/2056

Ocaña, G. (2012). Robótica como Asignatura en Enseñanza Secundaria. Resultados de una Experiencia Educativa. Espiral. Cuadernos del Profesorado, 5 (10), 56-64. Recuperado http://www.cepcuevasolula.es/espiral/articulos/ESPIRAL_VOL_5_N_10_ART_5. pdf

Ocaña, G., Romero, I. M., Gil, F., y Codina, A. (2015). Implantación de la nueva asignatura " Robótica " en Enseñanza Secundaria y Bachillerato. Investigación En La Escuela, 7(87), 65-79.

Olmedilla, J. M. M. (2006). Profesorado de Secundaria y Calidad de la Educación: Un marco de opciones políticas para la formación y el desarrollo profesional docente. Profesorado. Revista de Currículum Y Formación Del Profesorado, 10(1), 21. Recuperado de http://recyt.fecyt.es/index.php/profesorado/article/view/42026

Olmo, J. L. (2015). Zondle en la clase de matemáticas. Sociedad de La Información. Recuperado de http://sociedadelainformacion.com/56/jose1.pdf

Ovallos, D., Villalobos, B., De la Hoz, S., y Maldonado, D. (2016). Gamificación para la gestión de la innovación a nivel organizacional. Una revisión del estado del arte. Espacios, 37(8), 2-18.

Okoli, C. y Schabram, K. (2010). A Guide to Conducting a Systematic Literature Review of Information Systems Research. Working Papers on Information Systems, 10(26), 1-51. http://doi.org/10.2139/ssrn.1954824

Peña, M. J. y Madrid, M. J. (2015). Propuestas de Innovación para la enseñanza de los números primos. Revista de Educación Matemática, 32(1), 67-74.

Piaget, J. (1967). Psicología y pedagogía. Barcelona: Crítica, 2001.

Pico, S. (2013). Formación TIC del profesorado para garantizar el éxito en la integración de la tecnología. Itaca: Revista de Filología, 4, 65-80. http://doi.org/10.14198/ITACA2013.4.03

Plomp, T. (2013). Educational Design Research: An Introduction. En Plomp, T., \& Nieveen, N. (eds.). Educational Design Research. Enschede: SLO.

Necesidades formativas del profesorado de Secundaria para la implementación de experiencias gamificadas en STEM. Mercedes Fuentes Hurtado y Juan González Martínez.

Página 22 de 25 
Prensky, M. (2005) Adopt and Adapt. En Edutopia. Recuperado de www.edutopia.org/magazine/dec05

Prieto, A. B., Chrobak, R. y Plaza, M. J. (2012). Integración de TICs, investigación y herramientas metacognitivas en la educación de ciencias y ambiental. Estudio de caso: Cambio climático y eventos extremos en Patagonia Norte. En: A. J. Cañas, J. D. Novak, J. Vanhear, Eds. Concept Maps: Theory, Methodology, Technology. Proc. of the Fifth Int. Conference on Concept Mapping, 3, p.114-117. Recuperado de http://cmc.ihmc.us/cmc2012papers/cmc2012-p145.pdf

Reeves, T. C., Herrington, J., y Oliver, R. (2005). Design Research: A Socially Responsible Approach to Instructional Technology Research in Higher Education. Journal of Computing in Higher Education, 16(2), 96-115. http://doi.org/10.1007/s11412-009-9063-y

Revuelta, M. et al (2015). Jugando con robots en el aula : iniciativa para incentivar el ingreso de alumnos de la escuela secundaria a carreras de Ingeniería. En $X$ Congreso de Tecnología en Educación \& Educación en Tecnología Jugando (pp. 3-8). Corrientes. Recuperado de http://sedici.unlp.edu.ar/handle/10915/48341

Rodríguez, F., y Santiago, R. (2015). Gamificación. Cómo motivar a tu alumnado y mejorar el clima en el aula. Barcelona: Grupo Océano.

Salinas, J., De Benito, B., y Lizana, A. (2014). Competencias docentes para los nuevos escenarios de aprendizaje. Revista Interuniversitaria de Formación Del Profesorado, 79, 145-163.

Sánchez-Aparicio, J.C. (2014). Videojuegos y gamificación para motivar en educación. Andalucíaeduca, 120, 6-8. Recuperado de http://www.andaluciaeduca.com/hemeroteca/ae_digital120.pdf

Saorin, J. L., et al. (2015). Blokify: Juego de modelado e impresión 3D en tableta digital para el aprendizaje de vistas normalizadas y perspectiva. Digital Education Review, 27, 105-121. Recuperado de http://revistes.ub.edu/index.php/der/article/view/11651

Stohlmann, M., Moore, T. y Roehrig, G. (2012). Considerations for Teaching Integrated STEM Education. Journal of Pre-College Engineering Education Research. http://doi.org/10.5703/1288284314653

Tarabini, A., Curran, M., Montes, A., y Parcerisa, L. (2015). El Rol de los Centros Educativos en la prevención del Abandono Escolar. Una aproximación desde la perspectiva micropolítica. Educação, Sociedade \& Culturas, 45, 121-142.

Tauler, M. (2016). El Classcraft com a mesura per incentivar la motivació, l'atenció a la diversitat i l'aprenentatge de les ciencies naturals (Trabajo fin de máster). Universitat Pompeu Fabra de https://repositori.upf.edu/handle/10230/27061

Necesidades formativas del profesorado de Secundaria para la implementación de experiencias gamificadas en STEM. Mercedes Fuentes Hurtado y Juan González Martínez. 
Tobón, C. R. y Cano, O. R. (2015). Ambiente de aprendizaje basado en un robot móvil controlado con Kinect y Scratch. En Seminario Desarrollo tecnológico para la innovación educativa (pp. 1-11). Recuperado de http://reposital.cuaed.unam.mx:8080/jspui/handle/123456789/4590

Torres, S. (2016). Classcraft: una proposta de treball a l'aula. (Trabajo fin de máster). Universitat de les Illes Balears. Recuperado de http://dspace.uib.es/xmlui/handle/11201/1815

Tribó, G. (2008). El nuevo perfil profesional de los profesores de secundaria. Educación XXI, 11, 183-209. Recuperado de http://revistas.uned.es/index.php/educacionXX1/article/view/314/270

UNESCO. (2008). ICT competency standards for teachers: competency standards modules. Paris (France). Recuperado de http://portal.unesco.org/ci/en/ev.phpURL_ID=25731\&URL_DO=DO_TOPIC\&URL_SECTION=201.html

Urh, M., Vukovic, G., Jereb, E. y Pintar, R. (2015). The Model for Introduction of Gamification into E-learning in Higher Education. Procedia - Social and Behavioral Sciences, 197 (February), 388-397. Recuperado de http://doi.org/10.1016/j.sbspro.2015.07.154

Valle, D. (2016). Etoken: gamificación de la economía de fichas en el aula. (Trabajo fin de grado). Universidad de Sevilla, Sevilla. Recuperado de https://idus.us.es/xmlui/handle/11441/45061

Vázquez, A., y Manassero, M. A. (2009). Patrones actitudinales de la vocación científica y tecnológica en chicas y chicos de secundaria. Revista Iberoamericana de Educación, 50(4), 1. Recuperado de http://dialnet.unirioja.es/servlet/articulo?codigo=3055514\&info=resumen\&idioma $=\mathrm{SPA}$

Vázquez-Cano, E., y Ferrer, D. (2015). La creación de videojuegos con Scratch en educación secundaria. Communication Papers. Media Literacy \& Gender Studies, 4(6), 63-73. Recuperado de http://plataformarevistascomunicacion.org/2015/09/la-creacion-de-videojuegoscon-scratch-en-educacion-secundaria/

Vega, J., y Cañas, J. M. (2014). Curso de Robótica en Educación Secundaria usando Constructivismo Pedagógico. En Jornadas de Innovación y TIC Educativas JITICE'14. Universidad Rey Juan Carlos, Móstoles, Madrid. Recuperado de https://gsyc.urjc.es/jmplaza/papers/jitice2014.pdf

Vega-Moreno, D. et al. (2016). Integración de robótica educativa de bajo coste en el ámbito de la educación secundaria para fomentar el aprendizaje por proyectos. International Journal of Educational Research and Innovation (IJERI), 6, 162175. https://www.upo.es/revistas/index.php/IJERI/article/view/1653/1564 
Von Garnier, C. (2010). La metamorfosis necesaria en la escuela. Sevilla: Ituci siglo XXI.

Vygotsky, L. (1934). Pensamiento y lenguaje. Barcelona: Paidós Ibérica, 2010.

Werbach, K. (2012). Gamification. Coursera.org. Recuperado de https://www.coursera.org/course/gamification

Zabalza, M. A. (2003). Competencias docentes del profesorado universitario. Calidady desarrollo profesional. Madrid: Narcea.

Zichermann, G. y Cunningham, C. (2011). Gamification by Design: Implementing Game Mechanics in Web and Mobile Apps. Cambridge, MA: O'Reilly Media. 